\title{
Interview mit Regierungsrätin und SDK-Präsidentin Alice Scherrer
}

Frau Alice Scherrer ist 1947 geboren, verheiratet und Mutter von zwei Kindern. Der frauenrechtsmuffelige Kanton Appenzell-Ausserrhoden hat die Lehrerin an der Landsgemeinde 1994 zusammen mit einer weiteren Frau in den Regierungsrat gewählt, wo sie seither dem Gesundheitsdepartement vorsteht. Seit 1996 ist Frau Scherrer im Vorstand der Sanitätsdirektorenkonferenz SDK, der sie im Mai 2000 zur Präsidentin gewählt hat.

\section{Schweizerische Ärztezeitung:}

War es schwierig, sich in der männerdominierten Politik durchzusetzen?

Alice Scherrer: Nein, weder im Regierungsrat noch in den übrigen Gremien. Allfällige «Trennlinien» verlaufen weniger der Geschlechtergrenze entlang, sondern liegen in unterschiedlichen Werthaltungen.

Allerdings fühlte ich mich nach meiner Wahl verpflichtet, anderen Frauen den Weg zu ebnen, sei es beruflich oder politisch. Ich denke an die Verbesserung von Arbeitsbedingungen in Frauenberufen, an die Wahl von Frauen in Kaderpositionen (z. B. Verwaltungsleiterin, Mitglied Geschäftleitung Spitalverbund) und in politische Kommissionen.

\section{Haben die Politikerinnen als Minderheit Kontakte untereinander?}

Mir geht das «Minderheitsgefühl» völlig ab. Wohl deshalb empfinde ich die jährliche $\mathrm{Zu}$ sammenkunft von Exekutivfrauen aus Bund, Kantonen und Städten, die auf die Initiative von Bundesrätin Dreifuss zurückgeht, nicht als Minderheitentreffen sondern als Privileg. Es sind Events mit viel Gelegenheit zu Kontakten über Partei-, Sprach- und andere Grenzen hinweg. 2001 luden wir ehemalige Kolleginnen ein, um mit ihnen über die Zeit nach dem Rücktritt aus dem Amt - oder nach der Abwahl - zu sprechen. Es hat mich aufgewühlt, wie unterschiedlich die Abgangsmodalitäten in diesem kleinen Land sind und wie schwierig es offenbar ist, als Frau nach dem Amt wieder Fuss zu fassen. Da fehlen ganz einfach noch die Beziehungsnetze, um innert nützlicher Frist wieder eine befriedigende Funktion zu finden.
Ist der Föderalismus im Gesundheitswesen nicht ein Bremsklotz, der zu Gunsten grösserer Regionen abzubauen wäre?

Zum ersten Teil Ihrer Frage: Ich bin der Meinung, dass alles, was vor Ort entschieden und umgesetzt werden kann, auch dort getan werden soll. Föderalismus beinhaltet aber immer auch die Freiheit zur Zusammenarbeit mit denjenigen Partnern, mit denen ein Zusammengehen am erfolgversprechendsten ist.

Zum zweiten Teil Ihrer Frage eine Vorbemerkung: grössere Einheiten sind weder a priori effizienter noch kostengünstiger noch kundenfreundlicher - entscheidend ist, dass Organisation und Aufgabe kompatibel sind.

Wir Gesundheitsdirektoren und -direktorinnen arbeiten heute schon in vielen Bereichen vorbildlich zusammen - in unseren regionalen Konferenzen; in gemeinsamen Versorgungsregionen, die auf dem Vertragsweg entstehen und eine optimale Nutzung der Strukturen erlauben. Einige Aufgaben sind von den Kantonen auch an den Bund übergegangen.

Als Präsidentin der SDK würde ich mir gelegentlich wünschen, es wäre einfacher, als Kantone gemeinsame Positionen zu finden. Mir fehlt (noch) eine gemeinsame, eine anerkannte und respektierte Strategie von Bund und Kantonen im Hinblick auf die Gesundheit der Bevölkerung. Aber wir arbeiten bereits daran im Projekt «Nationale Gesundheitspolitik». Wir müssen uns auf eine gemeinsame Zielsetzung einigen, erst dann wird es möglich sein, das Unnötige vom Nötigen zu trennen, Prioritäten zu setzen - und den Spargriffel intelligent anzusetzen.

\section{Sind unter dem Finanzdruck der letzten Jahre} innovative Würfe überhaupt noch möglich?

Der Spielraum hat sich massiv verringert, wir verwenden heute den überwiegenden Teil unserer Arbeitszeit und unserer Energie darauf, die Budgets einzuhalten, das Aufwandwachstum einzudämmen. Die mühsamen Einsparungen durch die Straffung von Abläufen, von Stellenplänen und Leistungsangeboten werden jeweils gleich wieder aufgefressen durch Entscheide wie jenen des Versicherungsgerichts vom November 2001, der den Kantonen Mehrausgaben in Millionenhöhe beschert. 


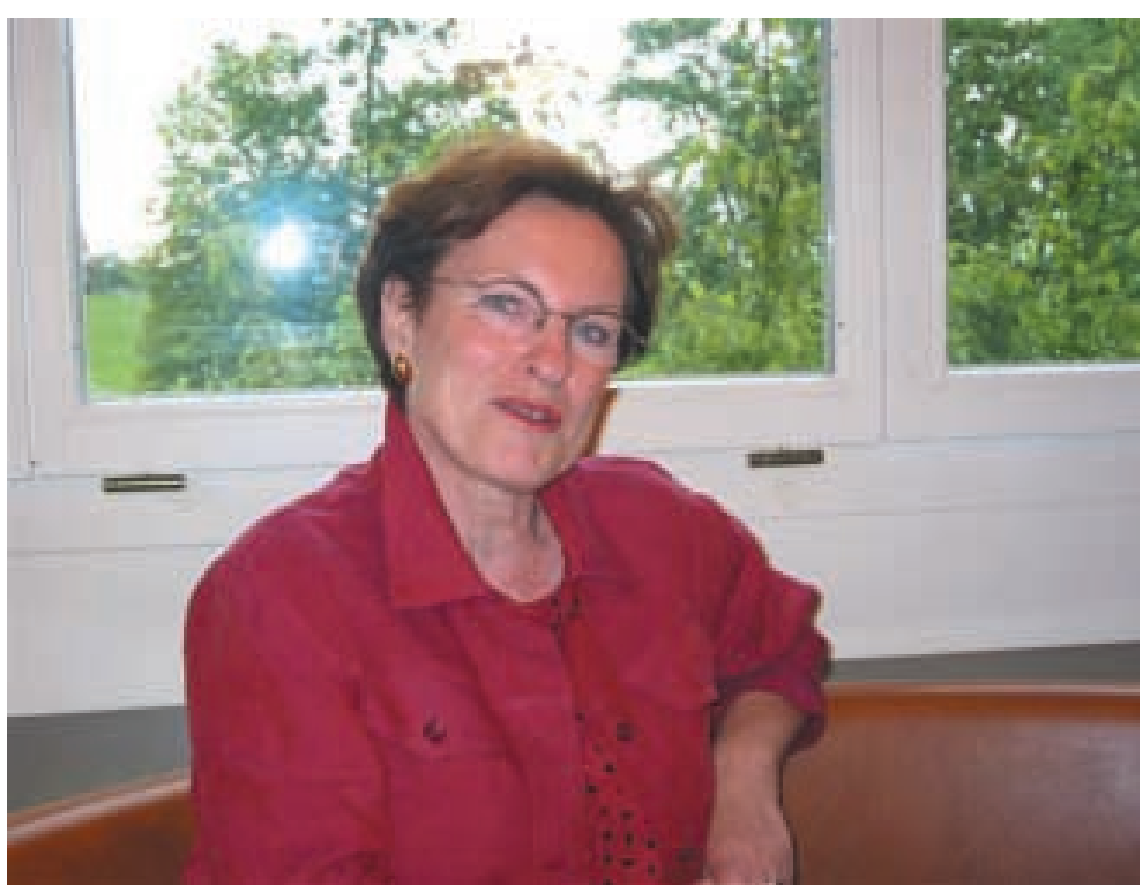

Der tägliche Druck droht den Blick auf die Zukunft zu verstellen. Man darf sich den Enthusiasmus nicht nehmen lassen, dann haben innovative Ideen immer noch eine Chance. Ich finde es absolut spannend, was sich heute im Bildungswesen Gesundheit tut, mit der Einpassung ins übrige Berufsbildungssystem, mit der neuen, direkt an die obligatorische Schulzeit anschliessenden Berufslehre zur/zum Fachangestellten Gesundheit.

Wie beurteilen Sie die Folgen der bilateralen Verträge für unser Gesundheitswesen?

Bilateral heisst gegenseitig - die Verträge eröffnen auch unseren Gesundheitsfachpersonen neue Möglichkeiten, das sollte man bei allen berechtigten - Bedenken nicht vergessen.

Wir befürchten, dass sich wiederholt, was im Fürstentum Liechtenstein passiert ist: dass sich die Gesundheitskosten nochmals massiv erhöhen durch EU-Ärztinnen und -Ärzte, die Praxen eröffnen. Wir kommen um Zulassungsbeschränkungen nicht herum. Die SDK unterstützt deshalb den Vorschlag des EDI für eine kurzfristige Begrenzung der Leistungserbringer als Notmassnahme. Die Nachteile - die eingeschränkten Berufschancen der jungen Ärzte und der zu erwartende administrative Aufwand sind uns bewusst. Persönlich zähle ich auch auf eine gewisse Solidarität und Verantwortung seitens der Leistungserbringer.
Sie setzen sich besonders für eine «Integrative Medizin» ein. Können Sie uns erklären, worum es sich dabei handelt?

Die Integrative Medizin baut die Brücke zwischen Schulmedizin und Naturheilkunde. Der Kanton Appenzell-Ausserrhoden hält in seinem Leitbild fest, dass er sich auf dem Hintergrund seiner traditionell liberalen Haltung im Bereich der Naturheilkunde als Dialogplatz für die Medizinrichtungen versteht. Der Mensch empfindet Gesundsein und Kranksein subjektiv. Er erwartet kompetentes und einfühlsames Eingehen auf seine individuelle Situation - wir zeigen auf, welche Antworten Schulmedizin und Naturheilkunde bereithalten.

\section{Kennen Sie ein Patentrezept gegen den stetig} wachsenden Aufwand im Gesundheitswesen? Leider nein. Es gibt weder schnelle noch einfache Rezepte, dafür ist das Gesundheitswesen, dafür ist unser eigenes Gesundheits- und Krankheitsverhalten zu komplex. Vermutlich hat nur das langfristige Zusammenspiel vieler Einzelschritte Erfolg. Das geht vom Umdenken bei jedem einzelnen über das Ausmerzen unanständiger Preispolitiken bis zur Beschränkung in der Zulassung von Leistungserbringern. Solange alle am «Gesundheitsmarkt» Beteiligten Jahr für Jahr nach höheren Erträgen streben, ein immer grösseres und feineres Leistungsangebot erwarten, dreht sich die Aufwandspirale - so einfach ist das. Mich stört zusätzlich, dass die Diskussion um die Gesundheitskosten unehrlich verläuft: man geisselt die Kosten und verschweigt geflissentlich, was diese über 40 Mia Franken jährlich für unsere Volkswirtschaft bedeuten. Die Gelder fliessen in Tausende von Lohntüten, halten die Pharmaund die Medizinaltechnikindustrie am Laufen, ermöglichen 100 verschiedene Krankenversicherungen, verwandeln sich in happige Dividenden, Verwaltungsratsentschädigungen und Beraterhonorare ... ergo wird eine wesentliche Kürzung des Aufwandes auch wesentliche Einbussen in diesen Bereichen bringen. Wer ist dazu bereit?

\section{Gibt es für Sie ein Land, das für Sie eine} Vorbildfunktion hat?

Ich nehme an den jährlichen Regionalkonferenzen der WHO für Europa teil. Ich sehe Länder, denen es ernster ist mit der Umsetzung ihrer gesundheitspolitischen Ziele als uns. 
Warum, so vermute ich wenigstens, lassen sich die meisten Volksvertreter in Privatkliniken behandeln?

Ich weiss nicht, ob Ihre Vermutung zutrifft, wenn es sich so verhält, ist dies ein schlechtes Signal. Ich halte die Dienstleistungen der öffentlichen Spitäler für ebenbürtig.

Für die Auswahl eines Spitals gibt es verschiedene Gründe: das Vertrauensverhältnis zu einem bestimmten Arzt, die geographische Nähe, die Anonymität, der Standard von Hotellerie und Infrastruktur, das «Markendenken»: es kann schick sein, in dieser oder jener Klinik zu liegen ...
Haben Sie noch einen speziellen Wunsch an unsere Leserinnen und Leser?

Ich wünsche mir, dass die Ärzteschaft nach den jahrelangen Diskussionen um TARMED sich wieder stärker engagiert in den eigentlichen Gesundheitsfragen. Zum Beispiel vermisse ich ihre Stimme, wenn es um die Beurteilung der Auswirkungen von (finanz- verkehrs-, bildungs-, wirtschafts-)politischen Entscheiden auf die Gesundheit der Bevölkerung geht.

Frau Regierungsrätin, ich danke Ihnen für das Gespräch.

Interview: Erhard Taverna 